\title{
The crossroads of anxiety: distinct neurophysiological maps for different symptomatic groups
}

\author{
This article was published in the following Dove Press journal: \\ Neuropsychiatric Disease and Treatment \\ 18 January 2016 \\ Number of times this article has been viewed
}

\section{Montserrat Gerez ${ }^{1-3}$ \\ Enrique Suárez ${ }^{2,3}$ \\ Carlos Serrano ${ }^{2,3}$ \\ Lauro Castanedo 2 \\ Armando Tello',3}

'Departamento de Neurofisiología Clínica, Hospital Español de México, Mexico City, Mexico; ${ }^{2}$ Departamento de Psiquiatría, Hospital Español de México, Mexico City, Mexico; ${ }^{3}$ Unidad de Postgrado, Universidad Nacional Autónoma de México, Mexico City, Mexico
Correspondence: Montserrat Gerez Departamento de Neurofisiología Clínica, Hospital Español de México, Ejército Nacional 6I3, DF II520, Mexico City, II 520 , Mexico

Tel +5252554960

Email mgerezm@hotmail.com
Background: Despite the devastating impact of anxiety disorders (ADs) worldwide, long-lasting debates on causes and remedies have not solved the clinician's puzzle: who should be treated and how? Psychiatric classifications conceptualize ADs as distinct entities, with strong support from neuroscience fields. Yet, comorbidity and pharmacological response suggest a single "serotonin dysfunction" dimension. Whether AD is one or several disorders goes beyond academic quarrels, and the distinction has therapeutic relevance. Addressing the underlying dysfunctions should improve treatment response. By its own nature, neurophysiology can be the best tool to address dysfunctional processes.

Purpose: To search for neurophysiological dysfunctions and differences among panic disorder (PD), agoraphobia-social-specific phobia, obsessive-compulsive disorder (OCD) and generalized anxiety disorder.

Methods: A sample population of 192 unmedicated patients and 30 aged-matched controls partook in this study. Hypothesis-related neurophysiological variables were combined into ten independent factors: 1) dysrhythmic patterns, 2) delta, 3) theta, 4) alpha, 5) beta (whole-head absolute power $z$-scores), 6) event-related potential (ERP) combined latency, 7) ERP combined amplitude (z-scores), 8) magnitude, 9) site, and 10) site of hyperactive networks. Combining single variables into representative factors was necessary because, as in all real-life phenomena, the complexity of interactive processes cannot be addressed through single variables and the multiplicity of potentially implicated variables would demand an extremely large sample size for statistical analysis.

Results: The nonparametric analysis correctly classified $81 \%$ of the sample. Dysrhythmic patterns, decreased delta, and increased beta differentiated AD from controls. Shorter ERP latencies were found in several individual patients, mostly from the OCD group. Hyperactivities were found at the right frontorbital-striatal network in OCD and at the panic circuit in PD.

Conclusions: Our findings support diffuse cortical instability in $\mathrm{AD}$ in general, with individual differences in information processing deficits and regional hyperactivities in OCD and PD. Study limitations and the rationale behind the variable selection and combination strategy will be discussed before addressing the therapeutic implications of our findings.

Keywords: anxiety disorders, dysrhythmic, epileptiform, ERP, EEG, LORETA

\section{Introduction}

Anxiety disorders (ADs) are the most prevalent and nearly the most pervasive mental health problems nowadays. ${ }^{1}$ Unresolved debates ${ }^{2}$ on whether the causes are biological, psychological, or social have moved into what happens first and how it ends up affecting the three realms. ${ }^{2}$ The academic quarrels have not answered the clinician's puzzle: who should be treated and how?

Treatment guidelines are based on nosological categories that classify each of the ADs as distinct entities., ${ }^{3,4}$ The distinctiveness has been supported by animal ${ }^{5}$ and 
human ${ }^{6}$ evidence of separate circuits mediating different forms of anxiety but is challenged by the high prevalence of comorbidity and longitudinal shifts. ${ }^{7}$ A single dimension, the "serotonin dysfunction disorders", ${ }^{8}$ was postulated based on the role of serotonin in animal fear models and the response to selective serotonin reuptake inhibitors (SSRIs). Neuroimaging supports the extended fear circuitry ${ }^{9}$ as the skeleton for all ADs, but also suggests additional networks that differ among subtypes. ${ }^{10}$ Subclassification has been suggested based on whether the symptoms are reactive or anticipatory. ${ }^{1-13}$ Accordingly, panic disorder (PD), agoraphobia-socialspecific phobia (SP) and obsessive-compulsive disorder (OCD) can be grouped as fear disorders, while generalized $\mathrm{AD}(\mathrm{GAD})$ and post-traumatic stress disorder (PTSD) belong to the distress disorder group. The distinction is supported by functional MRI (fMRI) studies showing different patterns of hyperactivation-inhibition in the structures of the extended fear circuit. ${ }^{13-15}$

Further separation has been suggested by a translational model in response to danger. ${ }^{16}$ Disorders with anticipatory anxiety (ie, GAD) are seen as the pathological correlate of exploring a dangerous environment, while the reactive disorders (PD, SP) as the result of a real or imagined confrontation with fearful stimuli. Because OCD and PTSD fall outside the evolutionary responses, they are grouped as a disorganized type. Separation of OCD from the other ADs is also suggested by a large body of neuroimaging and neuropsychological evidence on the involvement of regions other than the fear circuit, specifically, the extended orbito-frontostriatal network. ${ }^{17}$ Interaction between this network and the salience, the reward and the ventral attention networks is thought to participate in learning goal-directed behaviors. ${ }^{18}$ The structures in these networks are rich in dopamine, serotonin and glutamate receptors. Together, they coordinate a striatal facilitation of automatized sequences with a top-down prefrontal inhibition releasing the resources when a more adaptive response is available. Either orbito-fronto-striatal hyperactivity or poor prefrontal inhibition would result in various combinations of obsessive-compulsive, attentional, motivational, and ideo-motor symptoms.

The possibility that the dorsal-attention network may also be dysfunctional in ADs is suggested by clinical and psychophysiological evidence of a bidirectional relationship between attentional control and anxiety, ${ }^{19,20}$ as well as by comorbidity of AD with attention deficit across the lifespan. ${ }^{21}$ Altered connectivity among fear, affective and attentional networks in both $\mathrm{AD}^{22}$ and adult attention deficit disorder ${ }^{23}$ has been shown by fMRI studies.
Questioning whether anxiety is one or a combination of several disorders and whether it is caused by one or different types of dysfunction goes beyond conceptual frameworks. First-line treatments, SSRIs ${ }^{24}$ and cognitive-behavioral therapy, ${ }^{25}$ have shown adequate response rates. However, partial responses and recurrences are significant, and the rates differ across disorder subtypes. ${ }^{26}$ The progressive increase in the use of other drugs, particularly, antiepileptics, ${ }^{27}$ is only partially supported by controlled studies. To an unknown extent, it has been fostered by empirical exploration after repeated failures with treatments suggested by categorical guidelines. Finding the best strategy may take several trials and critical time.

The fastest way to a successful treatment is to address the physiopathogenic mechanisms. Attempts to unravel underlying mechanisms in ADs have been hampered by the dynamic complexity of the brain itself and by its job in coordinating links with internal and external environments. ${ }^{28}$ Complex systems are characterized by the emergence of new phenomena from higher-level interactions. These phenomena are necessarily atypical and do not follow pre-existing rules but a self-organizing principle that keeps the system balanced. ${ }^{29}$ Symptoms appear if the principle fails; the more complex the system, the harder it is to track the broken links. Metabolic conditions, personal history, and social context reflect on brain function to different extents, ${ }^{30}$ ultimately creating symptomatic individual differences. ${ }^{31}$ While strengthening compensatory mechanisms may suffice for some patients, others may require detangling the interacting processes to identify and target the dysfunctional ones.

Neurophysiology is a promising tool in such endeavor because its temporal resolution matches that of brain processes $^{32,33}$ and the spatial resolution has improved noticeably with source localization techniques. ${ }^{33-35}$ Several processes with distinct pathophysiological significance and, inherently, different therapeutic implications, can be studied during the same session or monitored sequentially. Also, it is non-invasive and widely accessible in clinical settings.

The neurophysiology literature on $\mathrm{AD}$ is extensive. Several surface electroencephalogram (sEEG) studies have reported a high prevalence of cerebral dysrhythmias in AD; for a review, see Shelley et al. ${ }^{36}$ Intracranial EEG has shown that at least some panic attacks ${ }^{37,38}$ and other anxiety episodes of longer duration ${ }^{39}$ can actually be focal epileptic seizures, even if only minor changes are seen in the sEEG. ${ }^{40}$ Paroxysmal activity has also been reported in intracranial recordings of OCD patients. ${ }^{41}$ Together with the well-known high association of obsessive-compulsive traits with focal 
epilepsy ${ }^{42}$ and the consistent finding of hyperactive frontostratial networks in OCD, ${ }^{43}$ these findings suggest shared mechanisms with epileptic neuronal populations.

Much of the recent neurophysiological work in AD has focused on background changes at rest or the response to specific stimuli. For an evidence-based medicine review, see Clark et al. ${ }^{44}$ Increased fast activity was reported by several studies in all AD groups and decreased slow activity was reported in OCD and phobias. Cortical instability is suggested by the majority of EEG studies across AD groups.

Several event-related potential (ERP) studies found decreased latencies in OCD and PD. Deficits in sensory gating were reported in OCD, PTSD, and phobias; enhanced responses to irrelevant stimuli; and impaired response inhibition in OCD and phobias. These findings may also relate to the functional unbalance at the orbito-fronto-striatal network discussed above. Despite the diversity of measurements and small sample sizes, Clark et $\mathrm{al}^{44}$ concluded that there was enough evidence to suggest task-related alterations in allocation of attentional resources with possible differences among disorder subtypes.

Three types of dysfunction emerge from the neurophysiological data: 1) hyperactive (epileptic-like) neuronal populations, 2) cortical instability, and 3) misallocation of attentional resources. Since brain function is a complex, self-regulating phenomenon, all three types may coexist as a combination of primary and compensatory mechanisms.

The general purpose of this work was to use neurophysiological measurements in search for dysfunctional patterns in AD. In particular, we wanted to know if the patterns were shared or distinctively related to $\mathrm{PD}, \mathrm{OCD}, \mathrm{SP}$ (including Social Phobia), and GAD.

\section{Methods Clinical groups}

The sample included 192 adult patients fulfilling the Diagnostic and Statistical Manual of Mental Disorders, 4th Edition,
Text Revision $(D S M-I V-\mathrm{TR})^{45}$ criteria for $\mathrm{AD}$ diagnosis and 30 age-matched controls. Exclusion criteria were: history of seizures, major perinatal event or head injury, left-handedness, current neurological disorder, another DSM-IV-TR Axis I diagnosis, and alcohol abuse or illegal drug use.

All were attended as outpatients, no one was suffering from a serious medical condition. To exclude comorbidity, patients fulfilling criteria for only one $D S M-I V$-TR AD diagnosis during or in the 6 months before the EEG recording were accepted. However, 73\% had some symptoms of another AD diagnosis, not enough to fulfill diagnostic criteria, and $45 \%$ had comorbidities with, or had switched from, another AD at an earlier time. First-time patients (n=97) were recorded before starting pharmacological treatment. Patients who were already medicated, but willing to participate, were further advised on the potential harm of the required 2-week withdrawal. Full awareness was mandatory. They were required to visit their attending psychiatrist at least twice a week for surveillance and support, and to remain at a close distance from facilities with 24-hour access to psychiatric services. No structured psychotherapy was added. Treatment reinstallation was necessary in 15 cases, excluded from the study. A total of 95 patients completed the 2-week offmedication period and were included in the study.

Patients were recruited prospectively over 10 years at the outpatient facilities of a major teaching hospital (Hospital Español de Mexico). Controls were recruited from family and friends of the hospital staff and by announcement through the radio, no monetary reward was offered. Additional exclusion criteria were: personal history of psychiatric disorder, and family history of epilepsy or hereditable psychiatric disorder. From 128 volunteers interviewed, only 30 fulfilled the inclusion criteria. (Table 1).

All subjects signed an informed consent form after detailed explanation of the purpose and conditions of the study. The project was approved and supervised by the Ethical Committee, Hospital Español de Mexico.

Table I Demographic characteristics and visual EEG findings of the sample based on the DSM-IV-TR criteria

\begin{tabular}{|c|c|c|c|c|c|c|c|c|}
\hline \multirow[t]{2}{*}{ Group } & \multirow[t]{2}{*}{ Subjects (n) } & \multicolumn{2}{|l|}{ Anti-anxiety drugs } & \multirow{2}{*}{$\frac{\text { Age (years) }}{\text { Mean (SD) }}$} & \multicolumn{2}{|l|}{ Sex } & \multicolumn{2}{|c|}{ Visual EEG finding } \\
\hline & & Never medicated & 2-weeks off medication & & Male & Female & Epileptiform & Marginal \\
\hline GAD & 53 & 31 & 22 & $34.3(13.6)$ & 27 & 26 & 7 (I3.2\%) & I 4 (26.4\%) \\
\hline OCD & 56 & 29 & 37 & $36.9(15.9)$ & 31 & 25 & II (I9.6\%) & $16(28.6 \%)$ \\
\hline PD & 38 & 16 & 22 & $30.8(10.9)$ & 14 & 24 & $8(21.0 \%)$ & $22(57.9 \%)$ \\
\hline SP & 45 & 31 & 14 & $34.2(9.9)$ & 21 & 24 & $6(13.3 \%)$ & $14(31.1 \%)$ \\
\hline Patients* & 192 & 107 & 95 & $34.1(13.4)$ & 93 & 99 & $32(16.7 \%)$ & $66(34.3 \%)$ \\
\hline CNTRL & 30 & NA & NA & $32.0(11.2)$ & 15 & 15 & $0(0 \%)$ & $2(6.7 \%)$ \\
\hline
\end{tabular}

Note: *The total number of subjects with a clinical diagnosis.

Abbreviations: CNTRL, control; DSM-IV-TR, Diagnostic and Statistical Manual of Mental Disorders, 4th Edition; EEG, electroencephalogram; GAD, generalized anxiety disorder; NA, not applicable; OCD, obsessive-compulsive disorder; PD, panic disorder; SD, standard deviation; SP, agoraphobia-social-specific phobia. 
Prior to recording, all subjects underwent unstructured psychiatric interview and semi-structured Anxiety Disorders Interview Schedule. ${ }^{46}$

\section{Neurophysiological recordings}

EEGs were recorded from 21 monopolar electrodes, international 10/20 system, ${ }^{47}$ linked-ears reference, impedance $<10 \mathrm{kOhms}$, electro-oculography, and electromyography control electrodes, using either Cadwell Spectrum 32 or NeuroScan Synamps, with sampling rate of $200 \mathrm{~Hz}$. Special care was taken to assure an eyes-closed, awake, and relaxed behavioral state.

Auditory ERPs, oddball paradigm,${ }^{48}$ were obtained with $20 \%$ rare/frequent $(1,500 / 1,000 \mathrm{~Hz})$ binaural stimuli, 1.5 mean inter-stimulus interval, eyes-open, center fixation, silent count.

\section{Neurophysiological measurements}

\section{$\mathrm{EEG}$, visual inspection}

Records were visually inspected by two certified electroencephalographers independently in order to identify dysrhythmic patterns. ${ }^{49}$ Records were identified by number to assure blindness about diagnosis, clinical conditions, and referral. Attended patterns were either epileptiform (spikes, spike-wave, polyspikes, and polyspike-wave) or marginal (midtemporal theta, wicket spikes, and 14-6/s discharges). These patterns have been traditionally considered normal variants because they are not related to epileptic syndromes. However, when observed, other non-epileptic dysfunctional conditions are present (ie, migraine, syncope, psychiatric disorders), and their relation to abnormal neuronal firing has been proposed by several authors. ${ }^{35,50}$

Inter-rater reliability was $85 \%$ for the classical epileptiform discharges vs marginal patterns. When in conflict, the final decision was taken after a joint discussion with a third electroencephalographer, usually with epilepsy training. Reliability was much lower for differences between "marginal" and normal patterns. Since these patterns are poorly defined and controverted in the literature, any disagreement was voted in favor of normal pattern. By this criterion, 31 dubitable records were declared normal.

\section{Quantitative EEG analysis}

Forty-eight 2.5 second EEG epochs were collected, carefully discarding artifacts or paroxysms, maintaining the sample reliability above $95 \%$ (ratio of variance between the even and odd seconds of the time series), resampled to $100 \mathrm{~Hz}$ and averaged before further analysis with NeuroGuide Software to obtain the z-transformed absolute power for each conventional band at each electrode site. ${ }^{51}$

\section{Event-related potential}

Latency and amplitude of the N100, N200, and P300 responses ${ }^{48}$ were measured ${ }^{34}$ at maximal negative peaks at Fz, the positive peak at Pz, time window 260-400 ms. After $\log$ transformation, $z$-scores were calculated against the laboratory database.

\section{Low-resolution electromagnetic topography}

Current source densities in 3D space were calculated with low-resolution electromagnetic topography (LORETA) method using the Key Institute software ${ }^{52}$ according to Talairach Atlas coordinates of the Montreal Neurological Institute's MRI. Cross-spectral values at each $1 \mathrm{~Hz}$ frequency band were multiplied by the $\mathrm{T}$ matrix $(x, y$, and $z$ current source moments in each of the 2,394 gray matter voxels, containing the corresponding weights for tissue connectivity in a 3-sphere model and Laplacian smoothing). The current sources of all voxels in a given region of interest (ROI) were averaged to create 66 current source densities, for each $1 \mathrm{~Hz}$ band, for each 2.5 second epoch. For details on theoretical grounds and mathematical transformations, see Gomez and Thatcher. ${ }^{53}$

The raw LORETA scores of the 48 epochs were averaged before further transformation. The 66 ROIs were reduced to 46 by combining adjacent ROIs with small number or voxels into ROIs with sufficient number approximate normal distribution for $z$ transformation with the NeuroGuide database. ${ }^{54,55}$

\section{Hypothesis-driven variable selection and hypothesis-guided combination}

From the widely varied symptoms and longitudinal courses in $\mathrm{AD}$, we expected several interacting processes weighted differently in each subject, but similar enough to create consistent patterns for similar symptoms. Therefore, we moved beyond single measures to look for combinations that could reflect the triad of frameworks.

Variable selection was based on 1) reliability and stability under the standardized conditions routinely used in our laboratory, and 2) known functional significance and possible relation to one of the three neurophysiological frameworks for AD.

Too many variables fulfilled these criteria; further reduction was achieved by either using previously reported combinations or combining under hypothesis guidance. 


\section{Variables related to cortical stability}

Whole-head absolute power $z$-scores of delta (WHDP), theta (WHTP), alpha and beta (WHBP) were obtained by averaging the $z$-scores of the 19 electrode sites. These measurements have been extensively used to study cortical stability and neurotransmitter balance ${ }^{56,57}$ and were included as quantitative factors.

\section{Variables related to attentional resource allocation}

From several ERP paradigms available, the auditory P300 was selected because of practical and theoretical reasons. Under standardized conditions, the measurements were highly reliable and the procedure was well known to the laboratory staff. It was designed to study the availability ${ }^{48}$ and allocation ${ }^{58}$ of attentional resources, and has been related to dopamine function in the orbito-fronto-striatal network. ${ }^{59}$ Various components of the $\mathrm{P} 300$ response reflect different stages of stimulus-driven (bottom-up) attentional processes. ${ }^{58} \mathrm{~A}$ more comprehensive ERP interpretation suggests top-down cortical control over the stimulus-response so that the registered waves are the final output of feedforward anticipation and feedback performance loops. ${ }^{60}$ Abnormal latencies and amplitudes of one or more components have been reported in $\mathrm{AD},{ }^{44}$ but the results differ regarding the affected ERP component. ${ }^{44}$ Besides the methodological differences, it is possible that $\mathrm{AD}$ patients have actually more inter- and intra-subject variability on the stages affected during attentional processes. Top-down modulation of the ERP predicts wider inter-individual variability because the response can be influenced by current context as well as by personal history. ${ }^{60} \mathrm{~A}$ more variable response would be predicted from altered dopaminergic (DA) function at the orbito-fronto-striatal network. ${ }^{59}$ Intending to address attentional processes regardless of specific stage, we studied central conduction velocity combining the latency $z$-scores of the N100, N200, and P300 components and the amount of allocated attentional resources by combining amplitude $z$-scores of the same three components.

\section{Variables related to hyperactive neuronal populations} Epileptic neuronal behavior is the oldest ${ }^{61}$ and the most elusive ${ }^{62}$ neurophysiological hypothesis in $\mathrm{AD}$. Its hallmark, epileptiform activity ${ }^{63}$ was included as a qualitative factor (EPI) with three levels (epileptiform, marginal, and none). The hypothesis of an epileptic neuronal behavior underlying $\mathrm{AD}$ has two main heuristic obstacles. One is the deep location of potentially relevant structures,${ }^{39,41}$ limiting the validity of negative findings on the sEEG. ${ }^{37}$ The other is the complexity of the phenomena under study. ${ }^{2,64}$ The clinical manifestations of focal discharges are usually short and stereotyped ${ }^{65}$ The complex features of AD symptoms could more likely be caused by the impact of focal discharges on the emergent properties of the system's dynamic interactions. Short- and long-lasting dynamic changes would reflect in episodic or chronic manifestations of the network dysfunction.

To search for possible complex network dysfunction at deep locations, we chose the LORETA method because it can measure and compare the distribution strength of multiple sources ${ }^{66,67}$ and has been used to study neuronal networks. ${ }^{68}$

Current densities are continuously changing in an apparently random or semi-chaotic fashion because of myriad ongoing processes unknown to the observer. ${ }^{69}$ Similar to some fMRI studies, ${ }^{70,71}$ we averaged 48 epochs overtime to identify regions of persistent hyperactivity against a continuously changing background.

Hyperactive networks can be caused not only by epileptic firing, but also by overload of network-related tasks. ${ }^{72}$ To further support epileptic-like behavior underlying the hyperactivities, the 30 narrow-band $z$-LORETA scores were combined into one broadband score (bzLORETA) for each ROI (bzROI). Broadband power has been used in quantitative EEG to identify epileptic foci in the absence of visually detectable epileptiform discharges ${ }^{73}$ because the broadband reflects the mixture of wavelengths contained in the spikewave complex even if it occurs at a small scale.

We wanted to study the three networks that have been implicated in AD by clinical, neuropsychological, and fMRI studies (see "Introduction" section). To reduce the factor load, and given that networks are defined by co-activation of regions,${ }^{74}$ we selected and combined the highly correlated ROIs within each network. Pearson's correlations ${ }^{75}$ were calculated for all the ROIs belonging to each one of the following networks: extended orbito-fronto-striatal (fnROI), ${ }^{43}$ extended panic (pnROI), ${ }^{9}$ and dorsal attention ROI. ${ }^{76}$ ROIs within each network with correlations above 0.85 were averaged to create one right and one left network-representative nROI for the three networks. Included ROIs and the represented network are shown in Table 2.

The highest bzLORETA from the six nROIs was divided by the mean bzLORETA of all ROIs as a measure of network hyperactivity (nbzLORETA). This measure was included in the analysis as a quantitative factor. The nROI from which it was obtained and the side - left/right - were included as categorical factors. 
Table 2 Regions of interest combined by model-network correspondence

\begin{tabular}{lll}
\hline Model network & EEG-LORETA ROIs & Label \\
\hline Panic & Amygdala (parahippocampal, uncinate) & pnROI \\
& Hippocampus & \\
& Insula & \\
Frontostriatal & Anterior cingulate & fnROI \\
& Medial prefrontal gyrus & \\
& Extra-nuclear zone & \\
& Orbital and rectal gyri & dnROI \\
Dorsal attention & Paracentral gyrus & \\
& Precuneus (parietal, occipital) & \\
& Superior parietal gyrus &
\end{tabular}

Notes: First column: networks postulated by animal models and human fMRI studies for response to danger (panic), goal-directed learning and selecting strategies (frontostriatal), and orienting attention (dorsal attention); second column: the EEGcombination of LORETA ROls representing each network in this study (selection criteria: $>0.85$ correlation among network ROls); third column: labels of the representative ROI combinations.

Abbreviations: $d n$, dorsal attention; fn, extended orbito-frontal-striatal; pn, extended panic; EEG, electroencephalogram; fMRI, functional magnetic resonance imaging; LORETA, low-resolution electromagnetic topography; ROI, region of interest.

\section{Statistical analysis}

Contingency tables and correspondence analysis ${ }^{75}$ with chisquare tests were used to describe the sample distribution and relation to clinical groups of the three categorical factors: EPI, cROI and side (Figure 1; Table 3), Fisher linear discrimination for the quantitative ones. ${ }^{75}$

The relationships of all factors among them and with clinical groups were tested under a General Linear Model
(GLM) ${ }^{75}$ dummy-coding the categorical variables. ${ }^{77}$ Even if the individual factors were normally distributed, our combination of variables created a complex data set. The probabilistic neural network classifier (PNNC) ) $^{78,79}$ adjusts to the available data and does not require any a priori information about the population. By using the PNNC, we expected robust and reliable results despite the complexity of the data.

PNNC assigned the observations to one of five clinical groups by creating a non-parametric estimate of each group's density function at a desired location based on neighboring observations from that group. Training was performed by back-propagating weights of the node connections and iteratively adapting the errors generated at the output, until the performance reached a plateau. ${ }^{80}$ Performance is defined by the error between target outputs, and actual network outputs (mean square error). Once the PNNC is trained, it can be applied to new, unknown data (validation sample).

We first trained the model with half of the sample randomly selected, and validated with the remaining half. The reliability was tested by repeating the classification 100 times with bootstrap,${ }^{81}$ from which we obtained mean classification accuracy, deviations, and confidence intervals.

To check for possible differences introduced by having had medication in the past, we repeated the procedure using the never-medicated patients as training and the 2-week offmedication as validation sample. The PNNC is more sensitive
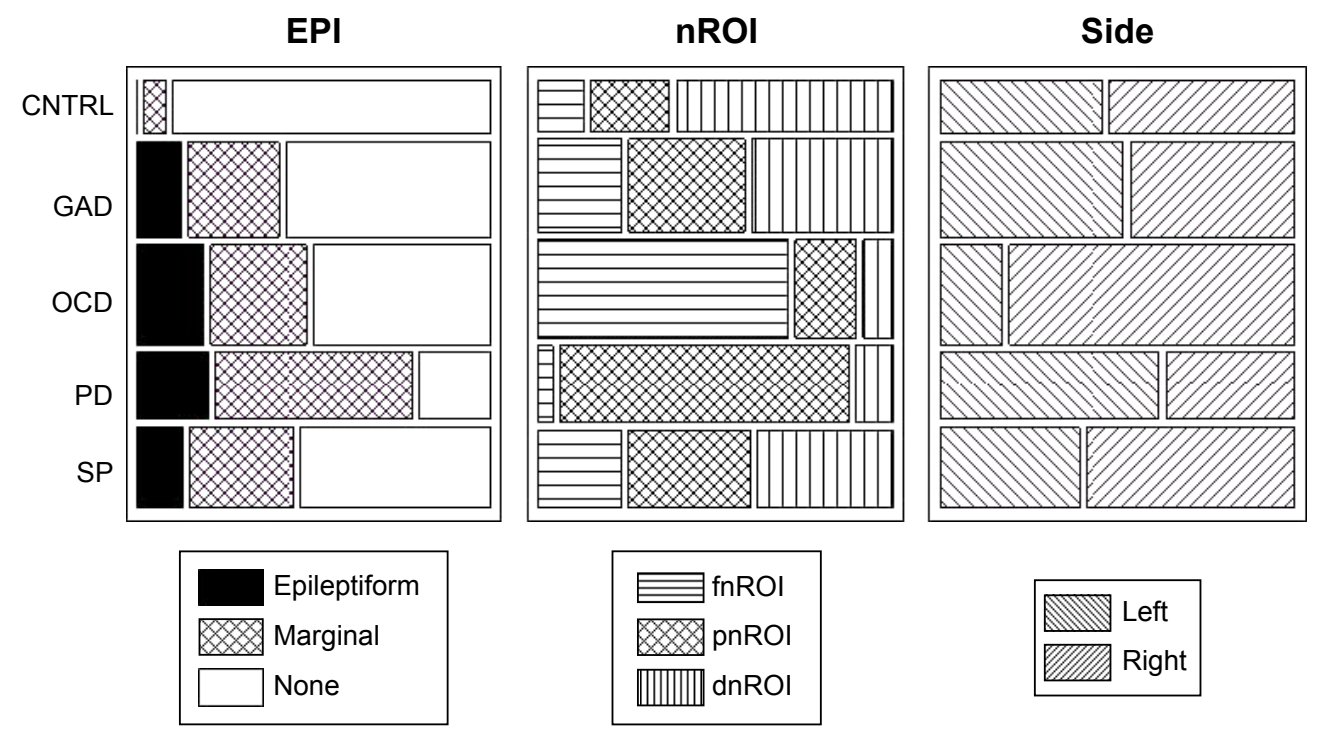

Figure I Multiple correspondence analysis.

Notes: Mosaic plots of the three categorical factors: dysrhythmic patterns (EPI) are shown in the box on the left, network (nROI) in the middle box, and side in the box on the right. Dysrhythmic patterns (EPI, left plot) were more frequent in the PD and OCD groups; hyperactive nROls (middle plot) from the frontostriatal network were more frequent in the OCD group, predominantly on the right side (right plot).

Abbreviations: CNTRL, control; dn, dorsal attention; EPI, epileptiform patterns at visual inspection; fn, extended orbito-fronto-striatal; GAD, generalized anxiety; OCD, obsessive-compulsive disorder; PD, panic disorder; pn, extended panic; nROI, network representative combination of ROIs; ROI, region of interest; SP, agoraphobia-socialspecific phobia. 
Table 3 Results from the ten single-factor linear discrimination with clinical group

\begin{tabular}{lllll}
\hline \multicolumn{6}{l}{ Relationship of each individual factor with clinical group } \\
\hline & Correlation & Total correct classification (\%) & Best group classification (\%) & Best classified group \\
\hline EPI & -0.15 & 26 & 74 & CNTRL \\
nROI & 0.14 & 33 & 75 & OCD \\
nbzLORETA & 0.19 & 34 & 57 & PD \\
Side (left/right) & 0.26 & 33 & 51 & OCD \\
WHDP & -0.41 & 35 & 76 & CNTRL \\
WHTP & 0.22 & 28 & 56 & SP \\
WHAP & 0.15 & 22 & 53 & CNTRL \\
WHBP & 0.32 & 42 & 61 & OCD \\
ERPI & 0.23 & 35 & 56 & PD \\
ERPa & 0.34 & 35 & 59 & PD
\end{tabular}

Notes: Correlations (second column) and classification rates (third column) were low for each of the factors when used alone to classify among the five groups. However, single factors showed fair discrimination rates (fourth column) for one group (fifth column) against all others: EPI and WHDP, independently, discriminated the CNTRL from others by negative relation to EPI; nROI discriminated the OCD group.

Abbreviations: CNTRL, control; EPI, epileptiform patterns at visual inspection; ERPI, z-scored combined latencies of the P300 components; ERPa, z-scored combined amplitudes of the P300 components; nROI, network representative combination of ROls; nbzLORETA, highest network-broadband z values of current source density; WHDP, z-scored whole head delta power; WHTP, z-scored whole head theta power; WHBP, z-scored whole head beta power; WHAP, z-scored whole head alpha power; LORETA, low-resolution electromagnetic topography; OCD, obsessive-compulsive disorder; PD, panic disorder; ROI, region of interest; SP, agoraphobia-social-specific phobia.

("unstable") than linear discriminant analysis or any other machine learning algorithm. Small changes in the training sample could cause large variations in the test results. ${ }^{80}$ Unknown information, whether random or patterned, would have affected classification accuracy.

Mean classification accuracy and standard deviations from the 100 bootstrap repetitions were used for the independent $t$-test between the two modes of defining the training sample.

\section{Results}

\section{General findings}

At the individual level, nearly all patients (190/192) showed atypical brain activity, whether by dysrhythmic patterns at visual inspection (98/192), significant deviations in quantitative measures (92/192), or both (178/192). Epileptiform activity was seen in 32 patients, marginal patterns in 66 patients, and two controls. Few small deviations in quantitative changes were seen for the control (CNTRL) group, not on the same subject, and not significantly affecting group averages.

Decreased WHDP $(z<-1.96, P<0.05)$ and increased WHBP $(z>1.96, P<0.05)$ were seen at the individual level in all patient groups but not in controls. Significantly shorter latency $z$-scores of the N100, N200, and P300 components and/or smaller amplitude of the event-related-potential were also found in $30 \%$ of the patients, not in controls. Longer latencies were one in the CNTRL and one in the PD groups.

Each of these findings, when used as single factor, could not predict group membership. The largest correlation with all groups was 0.41 for the WHDP. However, some single groups were fairly well classified by one of the factors: $74 \%$ of the controls were correctly identified by lacking dysrhythmic patterns, $76 \%$ by low deviations in WHDP, and $75 \%$ of OCD patients by more activity at the fnROI. The ten independently calculated Fisher's linear discriminations ${ }^{75}$ are shown in Table 3.

The $\mathrm{GLM}^{75}$ using all factors explained $77.4 \%$ of the variance ( $R$-squared corrected, $F=69.94, P=0.000$ ). Summary statistics of the multivariate analysis of the ten factors, function coefficients with confidence intervals and inflation factors below 10 for all factors are shown in Table 4. Inflation factors below 10 reflect no significant collinearities. A scatterplot of discriminant scores in 3D is shown in Figure 2, axes represented by the highest overall coefficients: bzLORETA, P300a, and WHBP. Interestingly, each of these measures came from a different neurophysiological hypothesis.

Chi-square decomposition of the correspondence analysis on the categorical factors (Table 5) showed significant contributions for CNTRL group from EPI = None (8.04/29.88), for the OCD group from the fnROI (38.17/116.24), for the right-side (6.30/17.21), and for the PD from the pnROI (22.08/116.18).

Mapping the group-means (Figure 3) showed decreased WHDP and increased WHBP in all clinical groups, but not in the controls. Increased zLORETA over the right orbitofronto-striatal and cingulate regions was seen in the OCD group, and over amygdala and insula in the PD group. These findings were bilateral as group-mean but lateralized in each subject. Group-mean maps were in close correspondence with predicted group profiles from the GLM coefficients: decreased WHDP, increased WHBP, and WHTP 
Table 4 Summary statistics for each of the seven quantitative and three categorical factors

\begin{tabular}{|c|c|c|c|c|c|c|c|c|}
\hline \multirow[t]{2}{*}{ Factor } & \multirow[t]{2}{*}{ Count } & \multirow[t]{2}{*}{ Mean } & \multirow{2}{*}{$\begin{array}{l}\text { Standard } \\
\text { deviation }\end{array}$} & \multicolumn{2}{|c|}{$\mathbf{9 5} \%$ confidence interval } & \multirow[t]{2}{*}{ Range } & \multirow{2}{*}{$\begin{array}{l}\text { Standardized } \\
\text { skewness }\end{array}$} & \multirow{2}{*}{$\begin{array}{l}\text { Standardized } \\
\text { kurtosis }\end{array}$} \\
\hline & & & & Minimum & Maximum & & & \\
\hline \multicolumn{9}{|l|}{ Quantitative } \\
\hline nbzLORETA & 222 & 1.18 & 0.48 & 0.13 & 2.20 & 2.07 & 1.04 & -1.94 \\
\hline ERPI & 222 & -0.05 & 0.86 & -2.25 & 1.88 & 4.14 & -0.87 & -1.89 \\
\hline ERPa & 222 & 0.05 & $0-97$ & -1.86 & 2.71 & 4.57 & 1.49 & -1.11 \\
\hline WHDP & 222 & -0.56 & 0.55 & -1.93 & 0.65 & 2.59 & -0.38 & -1.86 \\
\hline WHTP & 222 & 1.03 & 0.52 & -0.77 & 2.20 & 2.98 & -1.06 & -0.04 \\
\hline WHAP & 222 & -0.61 & 0.79 & -2.20 & 1.34 & 3.54 & 1.74 & $-|.5|$ \\
\hline WHBP & 222 & 1.32 & 0.74 & $-0.4 I$ & 2.93 & 3.34 & -1.73 & -1.56 \\
\hline \multicolumn{9}{|l|}{ Categorical } \\
\hline \multicolumn{9}{|l|}{$\mathrm{nROI}$} \\
\hline fnROI & 64 & 2.94 & 0.15 & 2.63 & 3.25 & NA & NA & NA \\
\hline pnROI & 89 & 2.39 & 0.13 & 2.14 & 2.64 & NA & NA & NA \\
\hline dnROI & 69 & 1.59 & 0.14 & 1.31 & 1.89 & NA & NA & NA \\
\hline \multicolumn{9}{|l|}{ Side } \\
\hline Left & 86 & 2.26 & 0.13 & 1.99 & 2.52 & NA & NA & NA \\
\hline Right & 136 & 2.36 & 0.11 & 2.16 & 2.57 & NA & NA & NA \\
\hline \multicolumn{9}{|l|}{ EPI } \\
\hline Epileptiform & 32 & 2.31 & 0.21 & 1.90 & 2.71 & NA & NA & NA \\
\hline Marginal & 68 & 2.46 & 0.14 & 2.18 & 2.74 & NA & NA & NA \\
\hline None & 122 & 2.16 & 0.10 & 1.95 & 2.36 & NA & NA & NA \\
\hline
\end{tabular}

Notes: Standardized skewness and kurtosis were below 2 for all quantitative factors, indicating no significant deviation from normal distribution.

Abbreviations: dn, dorsal attention; EPI, epileptiform patterns at visual inspection; ERPI, z-scored combined latencies of the P300 components; ERPa, z-scored combined amplitudes of the P300 components; fn, extended orbito-fronto-striatal; LORETA, low-resolution electromagnetic topography; NA, not applicable; nROI, network representative combination of ROls; pn, extended panic; ROI, region of interest; nbzLORETA, highest network-broadband $z$ values of current source density; WHDP, z-scored whole head delta power; WHTP, z-scored whole head theta power; WHBP, z-scored whole head beta power; WHAP, z-scored whole head alpha power.

in GAD; regional bzLORETA increase over the right fnROI, decreased WHDP, increased WHBP, and shorter P300 latency in OCD; increased bzLORETA at pnROI, no lateral predominance in PD; decreased WHDP, increased WHTP, and WHBP in SP.
Each subject showed different combination of findings but to some extent similar to the group profiles. As an example of individual differences within a group, Figure 4 shows findings of two OCD patients. At visual inspection, epileptiform activity was seen in one patient but not in

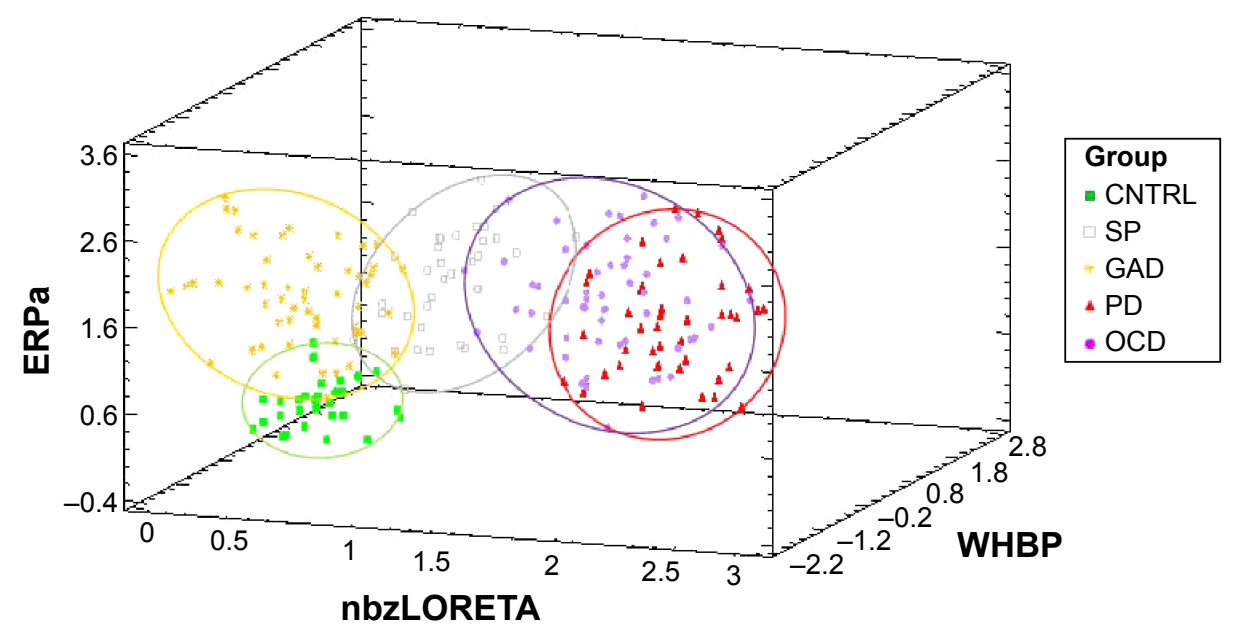

Figure 2 Probabilistic neural network classifier.

Notes: Scatterplot of the individual scores on the three measurements with highest discriminant weight: nbzLORETA, ERPa and WHBP. Segregation is clearer for the CNTRL (green), GAD (yellow), and SP (purple), with some overlap between the OCD (gray) and PD (red). These two groups showed more differences in nROI location and side, which are not plotted in this graph. Notice that each of these factors relates to one of the three hypothetical dysfunctions.

Abbreviations: CNTRL, control; ERPa, amplitude of the event-related-potentials; GAD, generalized anxiety disorder; nbzLORETA, highest network-broadband z values of current source density; nROI, network representative combination of ROls; OCD, obsessive-compulsive disorder; PD, panic disorder; SP, agoraphobia-social-specific phobia; WHBP, whole-head beta power. 
Table 5 Correspondence analysis of the three categorical factors (EPI, nROI, and side)

\begin{tabular}{|c|c|c|c|c|c|c|c|c|c|c|c|}
\hline \multirow{3}{*}{$\begin{array}{l}\text { Clinical } \\
\text { group }\end{array}$} & \multicolumn{11}{|c|}{ Chi-square distances } \\
\hline & \multicolumn{4}{|l|}{ EPI } & \multicolumn{4}{|l|}{ nROI } & \multicolumn{3}{|l|}{ Side } \\
\hline & None & Marginal & Epileptiform & Total & dnROI & fnROI & pnROI & Total & Right & Left & Total \\
\hline CNTRL & $8.04^{*}$ & 5.62 & 4.32 & 17.99 & 10.04 & 2.49 & 2.10 & 14.64 & 0.16 & 0.10 & 0.27 \\
\hline GAD & 0.28 & 0.31 & 0.05 & 0.64 & 1.85 & 0.34 & 0.49 & 2.69 & 2.72 & 1.72 & 4.43 \\
\hline OCD & 0.10 & 0.08 & 1.06 & 1.24 & 8.84 & $38.27 *$ & 6.90 & 54.01 & $6.30 * *$ & 3.99 & 10.29 \\
\hline PD & 3.83 & 4.89 & 0.35 & 9.08 & 5.77 & 9.28 & $22.08 *$ & 37.14 & 0.12 & 0.07 & 0.19 \\
\hline SP & 0.49 & 0.48 & 0.05 & 0.93 & 3.24 & $4.4 I$ & 0.04 & 7.69 & 1.24 & 0.79 & 2.031 \\
\hline Total & 12.65 & 11.39 & 5.84 & 29.88 & 29.75 & 54.80 & 31.62 & 116.18 & 10.54 & 6.67 & 17.21 \\
\hline
\end{tabular}

Notes: *Significant correspondence $(P<0.5)$ from the overall group centroid was found for $E P I=$ none in the $C N T R L$ group, $r R O I=$ fnROI for the OCD group, and pnROI for the PD group. **A high but not significant correspondence of side = right was found for OCD.

Abbreviations: CNTRL, control; dn, dorsal attention; EPI, epileptiform patterns at visual inspection; fn, extended orbito-fronto-striatal; GAD, generalized anxiety disorder; nROI, network representative combination of ROls; OCD, obsessive-compulsive disorder; PD, panic disorder; pn, extended panic; ROI, region of interest; SP, agoraphobiasocial-specific phobia.

other. Despite such a marked difference in the visual EEG, bzLORETA increases at right anterior regions and shorter P3001 were found in both patients.

The 100 bootstrap repetitions ${ }^{52}$ of the non-parametric discrimination $^{51}$ resulted in a mean discrimination accuracy of $80.95 \%$ for the randomly assigned training sample, $79.41 \%$ for the validation, $79.92 \%$ and $78.91 \%$ when the never-medicated patients entered as the training sample (for summary statistics refer to Table 6). The percentage of correctly classified subjects for each group is shown in Table 7. Groups showed different degrees of homogeneity in their neurophysiological patterns, the first discriminant function separates OD and PD from the other groups, the second discriminates all (Figure 5).

Classification accuracy did not differ between the two modes of running the PNNC: training sample constituted by never-medicated subjects, or, randomly selected $(t=1.29002$, $P=0.0198548$, 95\% confidence interval: -0.532141 , 2.54526).

\section{Discussion \\ Pitfalls and limitations}

The present work aimed to identify physiopathogenic mechanisms underlying ADs in general and possible differences related to symptomatic profiles. Some pitfalls were linked to the aim and will be addressed first.

\section{Sample composition}

We wanted to study a large sample of unmedicated patients in order to: 1) extract consistent neurophysiological patterns despite the wide variety of individual presentations in $\mathrm{AD}$ - the literature on neurophysiological findings in $\mathrm{AD}$ is extensive but inconclusive, mainly because of methodological differences and small sample sizes, ${ }^{44} 2$ ) avoid interference of anti-anxiety medications, known to modify neurophysiological measurements ${ }^{82}$ presumably by acting upon the same mechanisms we intend to investigate.

\section{Recruiting over a long time}

It took 10 years to recruit such a sample; meanwhile, there were changes in DSM, clinical scales and psychophysiological paradigms. Consistency was achieved by including only the type of data that were available from all subjects. Reclassification to $D S M-I V-\mathrm{TR}^{45}$ was possible because the Anxiety Disorders Interview Schedule ${ }^{46}$ scores included dimensional information, yet, it was not enough to match the $D S M-V^{5}$ criteria. All records included the $\mathrm{P} 300$ paradigm $^{49}$ and the resting EEG met the requirements to use the LORETA method. ${ }^{52}$

\section{Medication history}

The unmedicated condition introduced a bias because only half of the sample was completely naïve while the other was constituted by patients willing to participate, even if risking a withdrawal worsening. The majority of these had been poorly responsive to anxiolytic treatments. However, the impact of medication history was negligible, if any, on the analysis, since classification rates were not statistically different when the training sample was created from never-treated subjects when it was randomly selected $(t=1.29, P=0.02)$.

\section{Recruitment enviroment}

Another limitation of the study is that our sample may not represent the entire AD population. We asked the psychiatrists to refer all $\mathrm{AD}$ patients willing to participate regardless of symptoms severity. However, all the contacted psychiatrists work in a major teaching hospital, where the proportion of atypical or severe cases is usually higher than in community hospitals or primary care mental health facilities. 


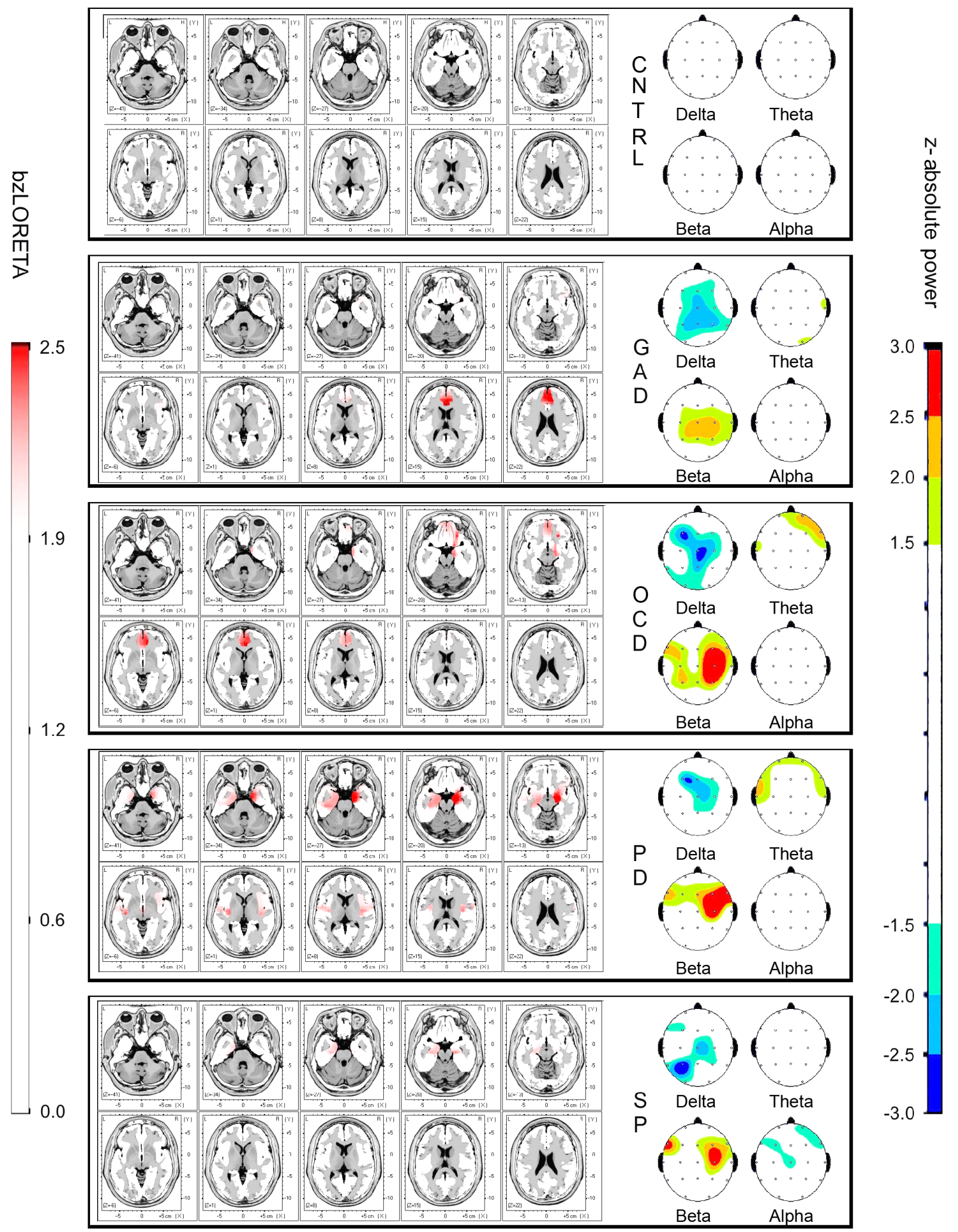

Figure 3 Group-mean maps of absolute power z-scores in the four conventional bands, and bzLORETA of the generalized anxiety disorder (GAD), obsessive-compulsive disorder (OCD), panic disorder (PD), and agoraphobia-social-specific phobia (SP) groups.

Notes: Decreased delta and increased beta were found in all groups. Focal changes in several bands were seen in the right frontal region of the OCD group and temporal regions in the PD group. bzLORETA was significantly increased at anterior cingulate in GAD, orbital and right extranuclear regions in OCD, bilateral amygdala and hippocampus and right insula in PD. Power z-scores on the right-side scale, bzLORETA scores on the left-side scale.

Abbreviations: bzLORETA, broadband z-transformed low-resolution electromagnetic topography; CNTRL, control. 
A

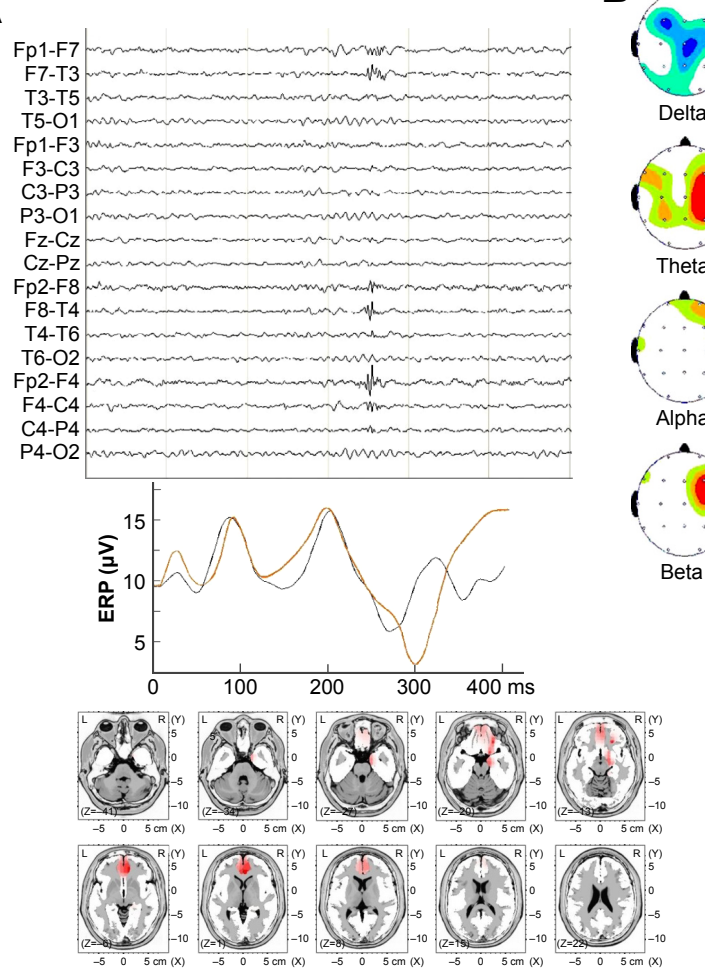

z-scores
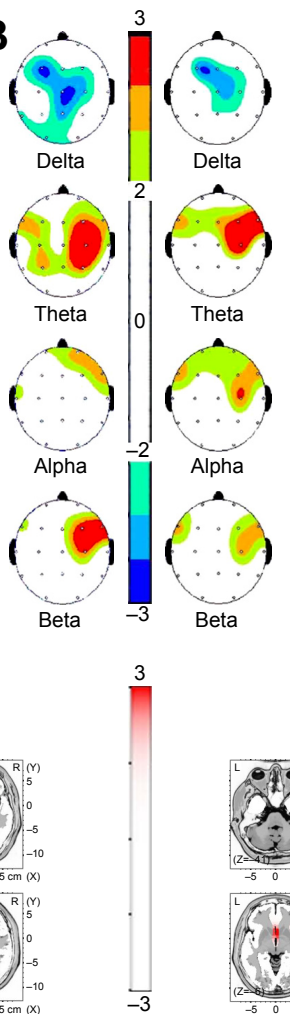

C

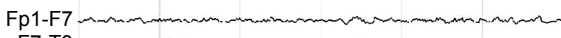

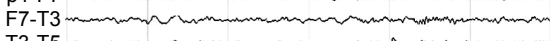
T3-T5 1 -O1

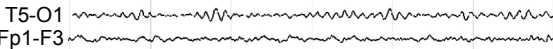

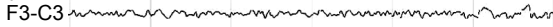
C3-P3 rn-wum

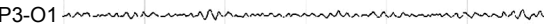
Fz-Cz FZ-CZ manm Cz-Pz F8-F8 ח

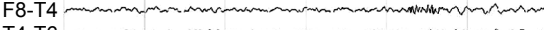
T4-T6 T6-O2

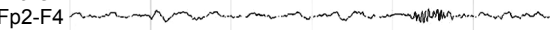
F4-C4 4 C4-P4 m P4-O2 - -
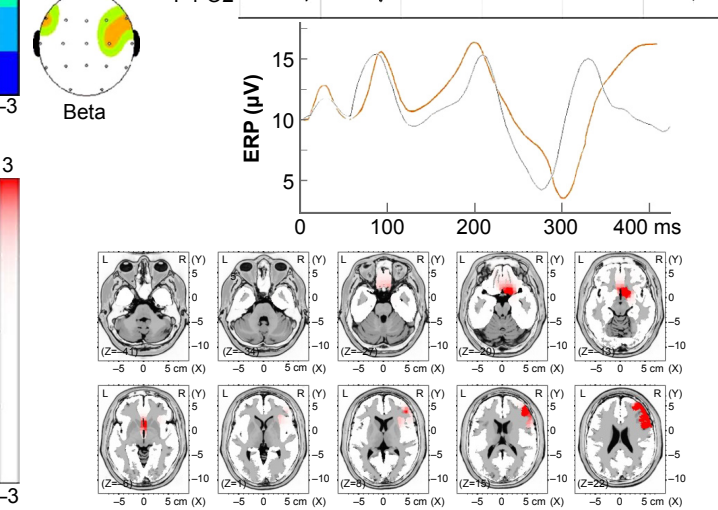

Figure 4 Individual examples of EEG trace (C), ERP, P300 response (B), absolute power (A), and bzLORETA maps (lower panels) of two patients with obsessive-compulsive disorder.

Notes: Epileptiform activity was seen in the EEG of one patient $(\mathbf{A})$ but not in the other $(\mathbf{C})$. Both showed power increases at more than two bands, earlier P300 response, and increased bzLORETA at orbital and right extranuclear regions.

Abbreviations: bzLORETA, broadband z-transformed low-resolution electromagnetic topography; EEG, electroencephalogram; ERP, event-related potential.

\section{Variable selection and combination}

The variety of symptom profiles, comorbidities and longitudinal switching in $\mathrm{AD}$ suggest dynamic interactions of multiple processes as underlying mechanisms. ${ }^{2}$ Single variables are insufficient to explore complex phenomena, ${ }^{83}$ and medical

Table 6 Classification results from the non-parametric discriminant function, Probabilistic Neural Network Classification (PNNC) for the training and validation samples, random-split

\begin{tabular}{llllll}
\hline \multirow{2}{*}{ Group } & \multicolumn{2}{l}{ Training sample } & & \multicolumn{2}{c}{ Validation sample } \\
\cline { 2 - 3 } & $\mathbf{N}$ & $\begin{array}{l}\text { Correctly } \\
\text { classified (\%) }\end{array}$ & & $\mathbf{N}$ & $\begin{array}{l}\text { Correctly } \\
\text { classified (\%) }\end{array}$ \\
\hline CNTRL & 15 & 99 & & 15 & 89 \\
GAD & 31 & 74 & & 22 & 71 \\
SP & 24 & 59 & & 14 & 59 \\
PD & 23 & 92 & & 22 & 89 \\
OCD & 19 & 87 & 37 & 87 \\
Total & 112 & 81 & 110 & 79 \\
\hline
\end{tabular}

Notes: A total correct classification of $81 \%$ was obtained during training of the PNNC with subjects selected by random split of the total sample, and $79 \%$ during validation with the remaining subjects.

Abbreviations: CNTRL, control; GAD, generalized anxiety disorder; N, number of subjects from each group; OCD, obsessive-compulsive disorder; PD, panic disorder; $\mathrm{SP}$, agoraphobia-social-specific phobia. problems are by definition complex. ${ }^{84,85}$ For centuries, the clinician's solution has been to combine all the available information from potentially implicated sources. Trying to adopt a clinician-like strategy, we were challenged by a large amount of neurophysiological information to select from, even if focusing only on hypothesis-related variables. Therefore, we combined those related to the same process and measured on the same scale, creating ten independent factors for the analysis. The statistical properties of each factor and its relation to the clinical group were investigated with descriptive and relational methods. Factor scores were normally distributed but, as often happens in the real world, homoscedasticity could not be assumed. ${ }^{75}$ The problem was addressed by using our final test as a non-parametric Bayesian discriminant model, ${ }^{78}$ the $\mathrm{PNNC}^{79}$ with 100 bootstrap repetitions to determine the confidence intervals of the classification accuracy. ${ }^{81}$

At this point, we shall emphasize that the discriminant function was not intended as a biomarker or a diagnostic test, but only to explore the potential use of combined information 
Table 7 Results from the 100 repetition bootstrap analysis on the classification rates obtained from the non-parametric discriminant function, Probabilistic Neural Network Classification on the whole sample divided by random-split into training and validation samples

\begin{tabular}{|c|c|c|c|c|c|c|}
\hline & CNTRL (\%) & GAD (\%) & SP (\%) & PD (\%) & OCD (\%) & Total (\%) \\
\hline \multicolumn{7}{|l|}{ Training sample } \\
\hline Mean correct classification & 98.91 & 74.42 & 59.50 & 92.45 & 87.30 & 80.95 \\
\hline Standard deviation & 3.00 & 9.73 & 11.67 & 5.89 & 6.45 & 3.63 \\
\hline Coefficient of variation & 3.04 & 13.08 & 19.61 & 6.37 & 7.39 & 4.48 \\
\hline Minimum & 84.61 & 47.62 & 37.04 & 73.33 & 72.41 & 70.69 \\
\hline Maximum & 100 & 95 & 77.78 & 100 & 100 & 86.21 \\
\hline Range & 15.38 & 47.38 & 40.74 & 26.67 & 27.58 & 15.52 \\
\hline Confidence interval upper & 99.76 & 77.19 & 62.82 & 94.12 & 89.13 & 81.98 \\
\hline Confidence interval lower & 98.061 & 71.66 & 56.18 & 90.78 & 85.46 & 79.92 \\
\hline \multicolumn{7}{|l|}{ Validation sample } \\
\hline Mean correct classification & 89.2 & 70.84 & 58.67 & 89.2 & 86.6 & 79.41 \\
\hline Standard deviation & 6.413 & 12.99 & 10.47 & 6.41 & 6.67 & 3.49 \\
\hline Coefficient of variation & 7.19 & 18.3 & 17.8 & 7.19 & 7.70 & 4.39 \\
\hline Minimum & 72.73 & 40 & 38.46 & 72.7 & 69 & 72.22 \\
\hline Maximum & 100 & 94.12 & 78.57 & 100 & 96.7 & 86.79 \\
\hline Range & 27.27 & 54.12 & 40.11 & 27.3 & 27.7 & 14.57 \\
\hline Confidence interval upper & 91.03 & 74.54 & 61.65 & 91 & 88.5 & 80.41 \\
\hline Confidence interval lower & 87.38 & 67.15 & 55.7 & 87.4 & 84.7 & 78.43 \\
\hline
\end{tabular}

Notes: Similar results were obtained from the 100 repetition bootstrap analysis with the "never-medicated" subjects as training sample and the "2-week off medication" as validation sample.

Abbreviations: CNTRL, control; GAD, generalized anxiety disorder; OCD, obsessive-compulsive disorder; PD, panic disorder; SP, agoraphobia-social-specific phobia.

when investigating complex behavioral phenomena. In that sense, despite sample and variable selection biases, oversimplification of measurements, and statistical limitations, the study fulfilled its purpose.

\section{General findings}

Nearly all patients showed evidence of atypical brain activity, varying in type and degree. Some dysfunctional mechanisms were common to all groups, with more or less individual differences, while others were related to diagnostic subtype. Dysrhythmic patterns at visual inspection, power increase in beta, and decrease in delta were frequent findings in individual patients from all clinical groups. Only two control subjects showed marginal patterns at visual inspection and one had increased ERP latencies; all other measures were within normal limits for the CNTRL group. Using single factor discriminators, this group obtained $76 \%$ correct classification with WHDP and $74 \%$ with EPI (negative correlation). Also,

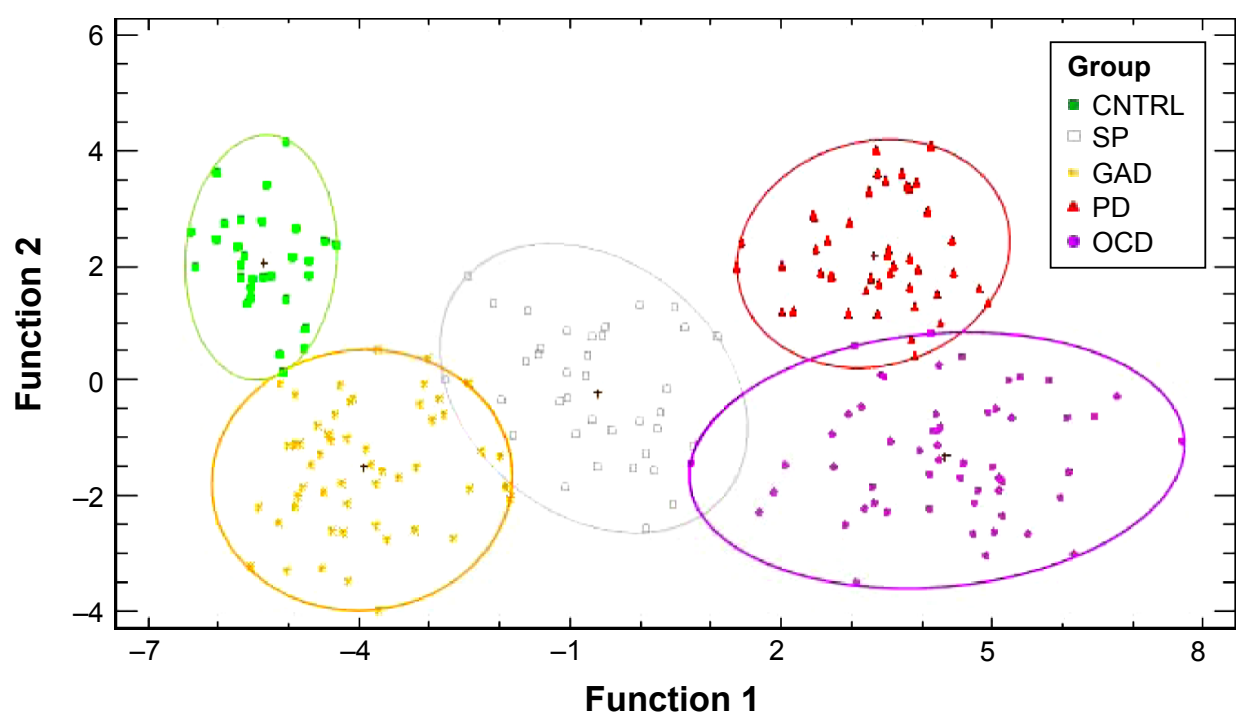

Figure 5 Scatterplot of vectors for the discriminant functions.

Note: The first derived function (Function I) separates the PD and OCD groups from all others while the second (Function 2) contributes to the definition among all. Abbreviations: CNTRL, control; GAD, generalized anxiety disorder; OCD, obsessive-compulsive disorder; PD, panic disorder; SP, agoraphobia-social-specific phobia. 
$75 \%$ correct classification of OCD patients was obtained with nROI, suggesting higher activation of a given network. Involvement of the right frontostriatal network was suggested by a significant association of OCD with fnROI and a nonsignificant with the right-side in the correspondence analysis of categorical factors. Another significant association was that of the PD group with the panic network.

Even though the controls were fairly well classified by not having dysrhythmic patterns or significant delta power deviations, none of these findings, when used as single-factor discriminant could predict overall group membership (highest correlation $=-0.41$, highest all-group classification $=42$ ) .

In contrast, the PNNC accurately classified $80 \%$ of the sample (Table 6). From this result, we conclude that complex phenomena are better explored by combining evidence from different sources.

\section{Hypothesis-related findings Cortical instability}

The most consistent findings across all AD groups were the whole-head power decrease in delta and increase in beta (Figure 3). These were the only relevant findings in GAD, suggesting that these changes are related to anxiety as a common symptom. Increased beta ${ }^{44}$ is one of the most consistent findings across AD studies. The functional significance of delta and beta activities has been under study since the beginning of the EEG. In wakefulness, beta activity denotes cortical activation, ${ }^{86}$ and low voltage delta oscillations are related to cognitive processing. ${ }^{87}$ Delta-beta coupling has been related to attentional control ${ }^{88}$ and efficiency of cognitive access. ${ }^{89}$ Our findings of whole-head power decreased delta and increased beta denote an uncoupled diffuse hyperactivation and cortical instability. Delta power has been related to brainstem serotonin activity. ${ }^{90,91}$ In animal models, ${ }^{90}$ different amygdaloid efferents to the hypothalamus and ventral tegmental area are involved in several types of anxiety-like responses. Brainstem serotonin neurons have efferent connections back to the amygdala and the neocortex, modulating their activity. Neuroimaging studies also support brainstem serotonin involvement in AD. ${ }^{91,92}$ It is possible that the good response seen in most AD patients, regardless of subtype, is mediated through the effect of increased brainstem serotonin activity.

\section{Misallocation of attentional resources}

Combined latency and/or amplitude of the ERP components were beyond $2 z$-scores in $30 \%$ of the patients, suggesting altered information processes. In the multivariate analysis, shorter latencies contributed to OCD group discrimination.
Despite methodological differences, OCD studies reported shortening of one or more of the component latencies. ${ }^{44}$ The role of dopamine on the generation of several ERP components has been thoroughly explored. ${ }^{59,93}$ Different lines of evidence suggest the role of DA in ERP latencies and amplitudes, as well as on the inter-trial variability, which in turn will affect the averaged amplitudes. Some of our patients, mostly from the OCD group, showed shorter latencies and increased amplitudes. This is opposite to what has been found in attention deficit patients who respond to DA medication. It is possible that the DA system plays a stronger role in the overall neurotransmitter deregulation in those patients with shortened ERP latencies.

\section{Network hyperexcitability}

The incidence of epileptiform activity (Table 1) was similar to that reported in other AD studies, ${ }^{30,44,50}$ but marginal patterns were more frequent in our patients. It is possible that our sample was biased toward atypical or more severe cases as discussed earlier. It is also possible that because "marginal patterns" lack a formal definition, we have included waveforms not considered by other studies. However, only two controls showed marginal patterns, the difference with clinical groups was large $(56.0 \%$ vs $6.7 \%)$ suggesting that indeed, those patterns have clinical implication and reflect some level of brain dysfunction.

The controls also differed from the clinical groups in not showing significant increases in bzLORETA, a factor included to identify hyperactive neuronal populations. In contrast, significant increases were seen at different locations in OCD, PD and SP (Figure 3). The increase was most consistent in the right fnROI for the OCD group, as shown by the correspondence analysis of categorical factors (Figure 1; Table 5). This is in agreement with studies from different neuroscience fields in OCD, ${ }^{17,41-43}$ further supported by evidence of functional hyperconnectivity at the orbito-fronto-striatal circuit. ${ }^{94}$

As discussed in the "Variables related to hyperactive neuronal populations" section, regional hyperactivities are not necessarily caused by epileptic discharges. Looking for epileptic-like changes we used broadband measures, the results turned out in favor. However, what actually supported the hypothesis of underlying epileptic behavior was the visual identification of epileptiform discharges at right frontal regions in several patients from the OCD group.

Hyperactivity at the panic network was suggested by the high bzLORETA scores in the PD group. Dysrhythmic patterns were also frequent among these patients. The main difference from the OCD group was regarding the location, 
affecting the panic network in PD group. Several sEEG have reported dysrhythmic patterns in PD and intracranial EEGs have shown ictal discharges during panic attacks. ${ }^{37-39}$ Yet, the relationship with mesiotemporal epilepsy remains elusive, ${ }^{95}$ not only because of the sEEG limitations addressed in the "Variables related to hyperactive neuronal populations" section, but also because of a relatively good initial response to SSRIs ${ }^{8}$ in contrast to the provocative, ${ }^{27,96,97}$ but not generalizable, ${ }^{98}$ response to antiepileptics.

The SSRI response is no longer an argument against the hypothesis of hyperactive (epileptic-like) neuronal populations underlying some of the AD symptoms. On the contrary, the antiepileptic effect of serotonin has been shown in animal models,${ }^{99}$ and its precursor, tryptophan, is currently under study in humans. ${ }^{100}$

Serotoninergic sensitization and subsequent kindling of the amygdala has been hypothesized as causal mechanism of illness progression in $\mathrm{AD} \cdot{ }^{101}$ On the other end, modulation of the hyperactive neurons has been proposed as the mechanism of action of SSRIs in anxiety and epileptic models, and presumably also in epilepsy patients. ${ }^{102}$ Accordingly, SSRIs could control anxiety symptoms even if caused by epileptic neuronal behavior. However, symptoms will reappear if the excessive discharge promotes the stage progression of amygdala kindling.

The conflicting reports on $\mathrm{AD}$ response to antiepileptics are difficult to evaluate because of methodological caveats. The presence or absence of cerebral dysrhythmias in potential responders has been ignored and few have addressed the mechanisms of action of the specific antiepileptic used. Nevertheless, some positive findings, together with the "unexplained" incremental trend in antiepileptic use in $\mathrm{AD},{ }^{24}$ suggest the potential advantage of this type of treatment, at least for a subset of patients.

Animal models show several types of long-lasting anxietylike behaviors induced by kindling amygdala circuits directly, through amygdalar stimulation, ${ }^{100}$ or indirectly by chronic stress. ${ }^{103}$ Not all kindled neurons result in clinical epilepsy, but the hyperactivity influences distally projecting zones, including other anxiety-related structures. The same process has been shown with intracranial recordings in humans, ${ }^{104}$ with evidence of diffuse cortical instability in the sEEG and chronic anxiety as clinical manifestation. Anxiety has also been suggested as a biomarker of epileptogenicity. ${ }^{105}$

The PD group had the lowest classification rates (59\%). A possible explanation would be that the underlying dysfunction would only appear in the presence of triggering stimuli, not included in our recording paradigm. However, only three subjects were misclassified as CNTRL, suggesting that even in the absence of stimuli-triggered symptoms, atypical patterns were present in their records. The other misclassified subjects were assigned to the PD and GAD groups. It is also possible that by including social phobia and agoraphobia the group was more heterogeneous. Patients with agoraphobia might have features more similar to the PD group, while those with social phobia could be related to GAD. The number was too small to test for these irregularities.

While most patients showed WHDP decrease or WHBP increase, regardless of subtype, not all showed dysrhythmic and/or bzLORETA increases. This suggests that in some patients, cortical instability may occur in the absence of network hyperactivity. A combination of dysfunctional mechanisms may explain different response patterns to SSRIs across the $\mathrm{AD}$ spectrum, ${ }^{24}$ as well as a marked improvement in some, but not all patients, by combining with antiepileptics. ${ }^{95-97}$

\section{Potential applications and future research}

Our findings suggest that neurophysiology can be used to identify ongoing dysfunctions, their relative weights and their interactive patterns on a moment-to-moment basis. Directly addressing ongoing dysfunctions should help in designing individual therapeutic strategies as well as developing dysfunction-targeted treatments. For example, good response to SSRIs alone may be expected if there is evidence of cortical instability only. Serotonin-dopamine modulators could be an alternative, if the DA-related ERPs are also affected. Dysrhythmic EEG with focal quantitative EEG changes may respond better to antiepileptics as adjunctive or stand-alone treatment, preferably those that are effective in the early phases of epileptogenesis.

Outlasting changes in networks behavior have been shown with psychotherapy. ${ }^{106-109}$ Understanding the dysfunctional patterns may accelerate functional restore. On the other hand, psychotherapy will always be necessary because, independently of the original cause and ongoing neuronal dysfunction, evidently the coping mechanisms of the $\mathrm{AD}$ patient are not working. Learning and holding on to more adaptive strategies should make the pharmacological response more successful and enduring.

Replication of these findings in wider, less biased samples of $\mathrm{AD}$ patients, and perhaps including question-related ERP paradigms may greatly enhance our understanding of physiopathogenic mechanisms. This, in turn will improve the design of therapeutic strategies. Treatment-response studies of neurophysiologically guided treatments are needed to build evidence-based guidelines. 


\section{Disclosure}

The authors report no conflicts of interest in this work.

\section{References}

1. Revicki DA, Travers K, Wyrwich KW, et al. Humanistic and economic burden of generalized anxiety disorder in North America and Europe. $J$ Affect Disord. 2012;140(2):103-112.

2. Bystritsky A, Khalsa SS, Cameron ME, Schiffman J. Current diagnosis and treatment of anxiety disorders. $P$ T. 2013;38(1):30-57.

3. World Health Organization. The ICD 10 Classification of Mental and Behavioral Disorders: Clinical Description and Diagnostic Guidelines. Geneva: World Health Organization; 1992.

4. American Psychiatric Association. Diagnostic and Statistical Manual for Mental Disorders. 5th ed. Washington, DC: American Psychiatric Association Press; 2013.

5. Lang PJ, Davis M, Ohman A. Fear and anxiety: animal models and human cognitive psychophysiology. J Affect Disord. 2000;61(3):137-159.

6. Heilman KM, Gilmore RL. Cortical influences in emotion. $J$ Clin Neurophysiol. 1998;15(5):409-423.

7. Vollebergh WA, Iedema J, Bijl RV, de Graaf R, Smit F, Ormel J. The structure and stability of common mental disorders: the NEMESIS study. Arch Gen Psychiatry. 2001;58(6):597-603.

8. Petty F, Davis LL, Kabel D, Kramer GL. Serotonin dysfunction disorders: a behavioral neurochemistry perspective. J Clin Psychiatry. 1996;57(Suppl 8):11-16.

9. Gorman JM, Kent JM, Sullivan GM, Coplan JD. Neuroanatomical hypothesis of panic disorder, revised. Am J Psychiatry. 2000;157(4):493-505.

10. Damsa C, Kosel M, Moussally J. Current status of brain imaging in anxiety disorders. Curr Opin Psychiatry. 2009;22(1):96-110.

11. Lang PJ, McTeague LM. The anxiety disorder spectrum: fear imagery, physiological reactivity, and differential diagnosis. Anxiety Stress Coping. 2009;22(1):5-25.

12. McTeague LM, Lang PJ. The anxiety spectrum and the reflex physiology of defense: from circumscribed fear to broad distress. Depress Anxiety. 2012;29(4):264-281.

13. Etkin A. Functional neuroanatomy of anxiety: a neural circuit perspective. Curr Top Behav Neurosci. 2010;2:251-277.

14. Shin LM, Liberzon I. The neurocircuitry of fear, stress, and anxiety disorders. Neuropsychopharmacology. 2010;35(1):169-191.

15. Duval ER, Javanbakht A, Liberzon I. Neural circuits in anxiety and stress disorders: a focused review. Ther Clin Risk Manag. 2015;11: 115-126.

16. Shuhama R, Del-Ben CM, Loureiro SR, Graeff FG. Animal defense strategies and anxiety disorders. An Acad Bras Cienc. 2007;79(1):97-109.

17. Menzies L, Chamberlain SR, Laird AR, Thelen SM, Sahakian BJ, Bullmore ET. Integrating evidence from neuroimaging and neuropsychological studies of obsessive-compulsive disorder: the orbitofronto-striatal model revisited. Neurosci Biobehav Rev. 2008;32(3):525-549.

18. Burguière E, Monteiro P, Mallet L, Feng G, Graybiel AM. Striatal circuits, habits, and implications for obsessive-compulsive disorder. Curr Opin Neurobiol. 2015;30:59-65.

19. Eysenck MW, Derakshan N, Santos R, Calvo MG. Anxiety and cognitive performance: attentional control theory. Emotion. 2007;7(2):336-353.

20. Berggren N, Derakshan N. Attentional control deficits in trait anxiety: why you see them and why you don't. Biol Psychol. 2013;92(3):440-446.

21. Abramovitch A, Dar R, Mittelman A, Wilhelm S. Comorbidity between attention deficit/hyperactivity disorder and obsessive-compulsive disorder across the lifespan: a systematic and critical review. Harv Rev Psychiatry. 2015;23(4):245-262.

22. Peterson A, Thome J, Frewen P, Lanius RA. Resting-state neuroimaging studies: a new way of identifying differences and similarities among the anxiety disorders? Can J Psychiatry. 2014;59(6):294-300.

23. McCarthy H, Skokauskas N, Mulligan A, et al. Attention network hypoconnectivity with default and affective network hyperconnectivity in adults diagnosed with attention-deficit/hyperactivity disorder in childhood. JAMA Psychiatry. 2013;70(12):1329-1337.
24. RavindranLN, Stein MB. The pharmacologic treatment of anxiety disorders: a review of progress. J Clin Psychiatry. 2010;71(7):839-854.

25. Hofmann SG, Asnaani A, Vonk IJ, Sawyer AT, Fang A. The efficacy of cognitive behavioral therapy: a review of meta-analyses. Cognit Ther Res. 2012;36(5):427-440.

26. Baldwin DS. Room for improvement in the pharmacological treatment of anxiety disorders. Curr Pharm Des. 2008;14(33):3482-3491.

27. Mula M, Pini S, Cassano GB. The role of anticonvulsant drugs in anxiety disorders: a critical review of the evidence. J Clin Psychopharmacol. 2007;27(3):263-272.

28. Andric M, Hasson U. Global features of functional brain networks change with contextual disorder. Neuroimage. 2015;117:103-113.

29. Hirsh JB, Mar RA, Peterson JB. Psychological entropy: a framework for understanding uncertainty-related anxiety. Psychol Rev. 2012; 119(2):304-320

30. Barlow DH. Unraveling the mysteries of anxiety and its disorders from the perspective of emotion theory. Am Psychol. 2000;55(11): $1247-1263$.

31. McEwen BS, Getz L. Lifetime experiences, the brain and personalized medicine: an integrative perspective. Metabolism. 2013;62 Suppl 1: S20-S26.

32. von Stein A, Sarnthein J. Different frequencies for different scales of cortical integration: from local gamma to long range alpha/theta synchronization. Int J Psychophysiol. 2000;38(3):301-313.

33. van Diessen E, Numan T, van Dellen E, et al. Opportunities and methodological challenges in EEG and MEG resting state functional brain network research. Clin Neurophysiol. 2015;126(8):1468-1481.

34. Lopes da Silva F. Functional localization of brain sources using EEG and/or MEG data: volume conductor and source models. Magn Reson Imaging. 2004;22(10):1533-1538.

35. Worrell GA, Lagerlund TD, Sharbrough FW, et al. Localization of the epileptic focus by low-resolution electromagnetic tomography in patients with a lesion demonstrated by MRI. Brain Topogr. 2000;12(4): 273-282.

36. Shelley BP, Trimble MR, Boutros NN. Electroencephalographic cerebral dysrhythmic abnormalities in the trinity of nonepileptic general population, neuropsychiatric, and neurobehavioral disorders. $J \mathrm{Neu}$ ropsychiatry Clin Neurosci. 2008;20(1):7-22.

37. Devinsky O, Sato S, Theodore WH, Porter RJ. Fear episodes due to limbic seizures with normal ictal scalp EEG: a subdural electrographic study. J Clin Psychiatry. 1989;50:28-30.

38. Sazgar M, Carlen PL, Wennberg R. Panic attack semiology in right temporal lobe epilepsy. Epileptic Disord. 2003;5:93-100.

39. Gerez M, Sada A, Tello A. Amygdalar hyperactivity, a fear-related link between panic disorder and mesiotemporal epilepsy. Clin EEG Neurosci. 2011;42(1):29-39.

40. Boulogne S, Catenoix H, Ryvlin P, Rheims S. Long-lasting seizurerelated anxiety in patients with temporal lobe epilepsy and comorbid psychiatric disorders. Epileptic Disord. 2015;17(3):340-344.

41. Amo C, Quesney LF, Ortiz T, et al. Limbic paroxysmal magnetoencephalographic activity in 12 obsessive-compulsive disorder patients: a new diagnostic finding. J Clin Psychiatry. 2004;65(2):154-162.

42. Kaplan PW. Obsessive-compulsive disorder in chronic epilepsy. Epilepsy Behav. 2011;22(3):428-432.

43. Melloni M, Urbistondo C, Sedeño L, Gelormini C, Kichic R, Ibanez A. The extended fronto-striatal model of obsessive-compulsive disorder: convergence from event-related potentials, neuropsychology and neuroimaging. Front Hum Neurosci. 2012;6:259.

44. Clark CR, Galletly CA, Ash DJ, Moores KA, Penrose RA, McFarlane AC. Evidence-based medicine evaluation of electrophysiological studies of the anxiety disorders. Clin EEG Neurosci. 2009;40(2):84-112.

45. American Psychiatric Association: Diagnostic and Statistical Manual of Mental Disorders. 4th ed. Text revision. Washington, DC: American Psychiatric Press; 2000.

46. Brown TA, Di Nardo PA, Barlow DH. Anxiety Disorder Interview Schedule for DSM-IV. San Antonio, TX: Psychological Corporation; 1994. 
47. Jasper HH. The ten twenty electrode system of the International Federation. Electroencephalogr Clin Neurophys. 1958;10:371.

48. Onofrj MC, Ghilardi MF, Fulgente T, et al. Mapping of event-related potentials to auditory and visual odd-ball paradigms. Electroencephalogr Clin Neurophys Suppl. 1990;41:183-201.

49. Stern JM, Engel J Jr. Atlas of EEG Patterns. Philadelphia, PA: Lippincott Williams and Wilkins; 2010.

50. Boutros N. Epileptiform discharges in psychiatric patients: a controversy in need of resurrection. Clin EEG Neurosci. 2009;40(4):239-244.

51. Thatcher RW. Validity and reliability of quantitative electroencephalography (qEEG). J. Neurother. 2010;14:122-152.

52. Pascual-Marqui RD, Michel CM, Lehmann D. Low resolution brain electromagnetic tomography: a new method for localizing electrical activity in the brain. Int J Psychophysiol. 1994;18:49-65.

53. Gomez JF, Thatcher RW. Frequency domain equivalence between potentials and currents using LORETA. Int J Neurosci. 2001;107(3-4): 161-171.

54. Thatcher RW, North D, Biver C. Evaluation and validity of a LORETA normative EEG database. Clin EEG Neurosci. 2005;36:116-122.

55. Thatcher RW, North D, Biver C. Parametric vs non-parametric statistics of low resolution electromagnetic tomography (LORETA). Clin EEG Neurosci. 2005;36(1):1-8.

56. Wright JJ, Robinson PA, Rennie CJ, et al. Toward an integrated continuum model of cerebral dynamics: the cerebral rhythms, synchronous oscillation and cortical stability. Biosystems. 2001;63(1-3):71-88.

57. Başar E, Başar-Eroğlu C, Güntekin B, Yener GG. Brain's alpha, beta, gamma, delta, and theta oscillations in neuropsychiatric diseases: proposal for biomarker strategies. Suppl Clin Neurophysiol. 2013;62: $19-54$.

58. Singhal A, Doerfling P, Fowler B. Effects of a dual task on the N100-P200 complex and the early and late Nd attention waveforms. Psychophysiology. 2002;39(2):236-245.

59. Pogarell O, Padberg F, Karch S, et al. Dopaminergic mechanisms of target detection - P300 event related potential and striatal dopamine. Psychiatry Res. 2011;194(3):212-218.

60. Kotchoubey B. Event-related potentials, cognition, and behavior: a biological approach. Neurosci Biobehav Rev. 2006;30(1):42-65.

61. Selling L. Hypothalamic epilepsy and anxiety states. Portland Clin Bull. 1951;5(1):5-12.

62. Adamaszek M, Olbrich S, Gallinat J. The diagnostic value of clinical EEG in detecting abnormal synchronicity in panic disorder. Clin EEG Neurosci. 2011;42(3):166-174.

63. Engel J. Report of the ILAE classification core group. Epilepsia. 2006;47(9):1558-1568.

64. Deco G, Jirsa VK, Robinson PA, Breakspear M, Friston K. The dynamic brain: from spiking neurons to neural masses and cortical fields. PLoS Comput Biol. 2008;4(8):e1000092.

65. Gloor P. Experiential phenomena of temporal lobe epilepsy. Facts and hypotheses. Brain. 1990;113( 6):1673-1694.

66. Lopes da Silva F. Functional localization of brain sources using EEG and/or MEG data: volume conductor and source models. Magn Reson Imaging. 2004;22(10):1533-1538.

67. Grech R, Cassar T, Muscat J, Camilleri KP, et al. Review on solving the inverse problem in EEG source analysis. J Neuroeng Rehabil. 2008;5:25.

68. Musso F, Brinkmeyer J, Mobascher A, Warbrick T, Winterer G. Spontaneous brain activity and EEG microstates. A novel EEG/fMRI analysis approach to explore resting-state networks. Neuroimage. 2010; 52(4):1149-1161.

69. Deco G, Jirsa VK, McIntosh AR. Emerging concepts for the dynamical organization of resting-state activity in the brain. Nat Rev Neurosci. 2011;12(1):43-56.

70. Darvas F, Pantazis D, Kucukaltun-Yildirim E, Leahy RM. Mapping human brain function with MEG and EEG: methods and validation. Neuroimage. 2004;23 Suppl 1:S289-S299.

71. He B. Modeling and Imaging of Bioelectric Activity: Principles and Applications. New York: Kluwer Academic/Plenum Publishers; 2004.
72. Woolgar A, Bor D, Duncan J. Global increase in task-related frontoparietal activity after focal frontal lobe lesion. J Cogn Neurosci. 2013; 25(9): 1542-1552.

73. Hughes JR, Taber JE, Fino JJ. The effect of spikes and spike-free epochs on topographic brain maps. Clin Electroencephalogr. 1991;22: $150-160$.

74. Raichle ME, MacLeod AM, Snyder AZ, Powers WJ, Gusnard DA, Shulman GL. A default mode of brain function. Proc Natl Acad Sci U S A. 2001;98(2):676-682.

75. Hays C. Statistics. 3rd ed. New York, NY: CBS College Publishing. 1981.

76. Petersen SE, Posner MI. The attention system of the human brain: 20 years after. Апnи Rev Neurosci. 2012;35:73-89.

77. Stockburger DW. Dummy coding. Multivariate Statistics: Concepts, Models, and Applications. Available from http://www.psychstat. missouristate.edu/multibook/mlt08m.html. Accessed December 28, 2013.

78. Lee HKH. Bayesian Nonparametrics via Neural Networks. Philadelphia, PA: SIAM; 2004.

79. Gish H. A probabilistic approach to the understanding and training of neural network classifiers. In: Proc. IEEE Int. Conf. Acoustic, Speech, Signal Processing. Albuquerque, NM: IEEE Press; 1990:1361-1364.

80. Brown DE, Corruble V, Pittard CL. A comparison of decision tree classifiers with backpropagation neural networks for multimodal classification problems. Pattern Recognit. 1993;26:953-961.

81. Efron B, Tibshirani RJ. Bootstrap methods for standard errors, confidence intervals and other measures of statistical accuracy. Stat Sci. 1986;1:54-77.

82. Saletu B, Grünberger J, Anderer P, Linzmayer L, Semlitsch HV, Magni G. Pharmacodynamics of venlafaxine evaluated by EEG brain mapping, psychometry and psychophysiology. Br J Clin Pharmacol. 1992;33(6):589-601.

83. Orsucci FF. The paradigm of complexity in clinical neurocognitive science. Neuroscientist. 2006;12(5):390-397.

84. Philippe P, Mansi O. Nonlinearity in the epidemiology of complex health and disease processes. Theor Med Bioeth. 1998;19(6):591-607.

85. Almeida Filho N. Towards a unified theory of health-disease: II. Holopathogenesis. Rev Saude Publica. 2014;48(2):192-205.

86. Jones BE. From waking to sleeping: neuronal and chemical substrates. Trends Pharmacol Sci. 2005;26(11):578-586.

87. Harmony $T$. The functional significance of delta oscillations in cognitive processing. Front Integr Neurosci. 2013;7:83.

88. Morillas-Romero A, Tortella-Feliu M, Bornas X, Putman P. Spontaneous EEG theta/beta ratio and delta-beta coupling in relation to attentional network functioning and self-reported attentional control. Cogn Affect Behav Neurosci. 2015;15(3):598-606.

89. Nakatani C, Raffone A, van Leeuwen C. Efficiency of conscious access improves with coupling of slow and fast neural oscillations. $J \operatorname{Cog} n$ Neurosci. 2014;26(5):1168-1179.

90. Griebel G. 5-Hydroxytryptamine-interacting drugs in animal models of anxiety disorders: more than 30 years of research. Pharmacol Ther. 1995;65(3):319-395.

91. Nikolaus S, Antke C, Beu M, Müller HW. Cortical GABA, striatal dopamine and midbrain serotonin as the key players in compulsive and anxiety disorders - results from in vivo imaging studies. Rev Neurosci. 2010;21(2):119-139.

92. Lowry CA, Johnson PL, Hay-Schmidt A, Mikkelsen J, Shekhar A. Modulation of anxiety circuits by serotonergic systems. Stress. 2005; $8(4): 233-246$

93. Polich J. Updating P300: an integrative theory of P3a and P3b. Clin Neurophysiol. 2007;118(10):2128-2148.

94. Beucke JC, Sepulcre J, Talukdar T, et al. Abnormally high degree connectivity of the orbitofrontal cortex in obsessive-compulsive disorder. JAMA Psychiatry. 2013;70(6):619-629.

95. Pande AC, Pollack MH, Crockatt J, et al. Placebo-controlled study of gabapentin treatment of panic disorder. J Clin Psychopharmacol. 2000; $20: 467-471$ 
96. Davis LL, Ryan W, Adinoff B, Petty F. Comprehensive review of the psychiatric uses of valproate. J Clin Psychopahrmacol. 2000;20(1) Suppl 1:1S-17S.

97. Hoffman EJ, Mathew SJ. Anxiety disorders: a comprehensive review of pharmacotherapies. Mt Sinai J Med. 2008;75(3):248-262.

98. Citraro R, Scicchitano F, De Fazio S, et al. Preclinical activity profile of $\alpha$-lactoalbumin, a whey protein rich in tryptophan, in rodent models of seizures and epilepsy. Epilepsy Res. 2011;95(1-2):60-69.

99. Mainardi P, Leonardi A, Albano C. Potentiation of brain serotonin activity may inhibit seizures, especially in drug-resistant epilepsy. Med Hypotheses. 2008;70(4):876-879.

100. Adamec R, Young B. Neuroplasticity in specific limbic system circuits may mediate specific kindling induced changes in animal affect implications for understanding anxiety associated with epilepsy. Neurosci Biobehav Rev. 2000;24(7):705-723.

101. Post RM, Weiss SR. Sensitization and kindling phenomena in mood, anxiety, and obsessive-compulsive disorders: the role of serotonergic mechanisms in illness progression. Biol Psychiatry. 1998;44(3):193-206.

102. Hamid H, Kanner AM. Should antidepressant drugs of the selective serotonin reuptake inhibitor family be tested as antiepileptic drugs? Epilepsy Behav. 2013;26(3):261-265.
103. Jones NC, O’Brien TJ. Stress, epilepsy, and psychiatric comorbidity: how can animal models inform the clinic? Epilepsy Behav. 2013;26(3): 363-369.

104. Tebartz van Elst L, Krishnamoorthy ES, Schulze-Bonhage A, et al. Local area network inhibition: a model of a potentially important paraepileptic pathomechanism in neuropsychiatric disorders. Epilepsy Behav. 2011;22(2):231-239.

105. Kanner AM, Mazarati A, Koepp M. Biomarkers of epileptogenesis: psychiatric comorbidities (?). Neurotherapeutics. 2014;11(2):358-372.

106. Beauregard M. Functional neuroimaging studies of the effects of psychotherapy. Dialogues Clin Neurosci. 2014;16(1):75-81.

107. Porto PR, Oliveira L, Mari J, Volchan E, Figueira I, Ventura P. Does cognitive behavioral therapy change the brain? A systematic review of neuroimaging in anxiety disorders. JNeuropsychiatry Clin Neurosci. 2009;21(2):114-125.

108. Barsaglini A, Sartori G, Benetti S, Pettersson-Yeo W, Mechelli A. The effects of psychotherapy on brain function: a systematic and critical review. Prog Neurobiol. 2014;114:1-14

109. Messina I, Sambin M, Palmieri A, Viviani R. Neural correlates of psychotherapy in anxiety and depression: a meta-analysis. PLoS One. 2013;8(9):e74657.
Neuropsychiatric Disease and Treatment

\section{Publish your work in this journal}

Neuropsychiatric Disease and Treatment is an international, peerreviewed journal of clinical therapeutics and pharmacology focusing on concise rapid reporting of clinical or pre-clinical studies on a range of neuropsychiatric and neurological disorders. This journal is indexed on PubMed Central, the 'PsycINFO' database and CAS,

\section{Dovepress}

and is the official journal of The International Neuropsychiatric Association (INA). The manuscript management system is completely online and includes a very quick and fair peer-review system, which is all easy to use. Visit http://www.dovepress.com/testimonials.php to read real quotes from published authors. 